\title{
An update on Wnt signaling pathway in cancer
}

\author{
Yanlu Zhang ${ }^{1 \#}$, Dan Zu ${ }^{1 \#}$, Zhe Chen ${ }^{2}$, Guoqing Ying ${ }^{1}$ \\ ${ }^{1}$ College of Pharmaceutical Science, Zhejiang University of Technology, Hangzhou 310014, China; ${ }^{2}$ First Clinical Medical College, Zhejiang Chinese \\ Medical University, Hangzhou 310053, China \\ Contributions: (I) Conception and design: D Zu, Y Zhang; (II) Administrative support: G Ying, Z Chen; (III) Provision of study materials or patients: \\ Y Zhang, G Ying, Z Chen; (IV) Collection and assembly of data: D Zu, Y Zhang; (V) Data analysis and interpretation: D Zu, Y Zhang; (VI) \\ Manuscript writing: All authors; (VII) Final approval of manuscript: All authors \\ \#These authors contributed equally to this work. \\ Correspondence to: Guoqing Ying. College of Pharmaceutical Science, Zhejiang University of Technology, Hangzhou 310014 , China. \\ Email: gqying@zjut.edu.cn; Zhe Chen. First Clinical Medical College, Zhejiang Chinese Medical University, Hangzhou 310014, China. \\ Email: chenzhe@zju.edu.cn.
}

\begin{abstract}
Wnt signaling involves many aspects of development, cell biology and physiology. Mutations in the Wnt gene can lead to abnormal embryonic development and cancer formation, including various aspects that affect proliferation, morphogenesis, and differentiation. The occurrence and development of tumors is a complex process involving multiple factors. The Wnt signaling pathway participates in this process as an anti-tumor target by activating multiple gene transcriptions. The emergence of Wnt pathway inhibitors and targeted drugs has opened up a new world of cancer treatment. This review focuses on the mechanism of action of the Wnt signaling pathway in different cancers. Secondly, we have organized and introduced the latest Wnt anti-tumor drugs.
\end{abstract}

Keywords: Wnt signaling pathway; $\beta$-catenin; inhibitor; cancer

Submitted Nov 01, 2019. Accepted for publication Dec 03, 2019.

doi: $10.21037 /$ tcr.2019.12.50

View this article at: http://dx.doi.org/10.21037/tcr.2019.12.50

\section{Introduction}

The Wnt gene plays a role in the normal embryonic development of mice. It controls the axial development of the embryo (1). The Wnt signaling pathway is critical for the regulation of cell proliferation, differentiation, apoptosis and migration. Mutations in the Wnt gene or Wnt pathway components can lead to abnormal embryonic development and cancer formation, including cell proliferation, differentiation and metastasis (2). With the continuous development of tumor biology, it has been found that the Wnt pathway is abnormally activated in tumor cells, mainly in three aspects: proteins and transcription factors that make up the Wnt pathway are destroyed; more Wnt signal makes the pathway active and cells proliferate excessively; other factors in the cell stimulate the cells to produce abnormal reactions through the Wnt pathway. In addition, mutations in different proportions of $\beta$-catenin nuclei and different frequencies of beta-catenin gene (CTNNB)/adenomatous polyposis coli (APC) were detected in many cancers (3). Therefore, various targeted drugs for mutation targets are constantly emerging (4).

\section{Canonic Wnt signal pathway}

The Wnt protein forms a trimer on the cell surface by completing with the Frizzled/low-density lipoprotein (LDL) receptor-associated protein (LRP) (5). The binding of Wnts to frizzle (FZD) and LRP5 or LRP6 co-receptors transduces a signal across the plasma membrane that results in the activation of dishevelled protein. Activation of disheveled (DVL) prevents APC, AXIN and glycogen synthase kinase 3 (GSK3 $\beta$ ) from forming a disruptive complex, which prevents the phosphorylation and subsequent degradation of $\beta$-catenin, and accumulates in the cytoplasm or translocates to the nucleus (6). Once inside the nucleus, $\beta$-catenin 
replaces groucho with the transcription factor $\mathrm{T}$-cell factor (TCF)/lymphoid enhancer-binding factor (LEF) binding transcriptional activation complex and recruit histone acetylases. This leads to the transcriptional activation of growth promoting genes such as cyclooxygenase2, matrilysin/matrix metalloproteinase 7, cycling D1 (7). In the absence of specific Wnt ligands, the Wnt receptor failed to bind the dishevelled protein, and the cytoplasmic $\beta$-catenin was phosphorescent by the disrupted complex formed by the three proteins APC, AXIN and GSK3 $\beta$. The initial casein kinase 1 (CKI) phosphorylation occurs at Ser45, which in turn primes the molecule by phosphorylation of GSK3 $\beta$ on Thr41, Ser37 and Ser3 (7). Phosphorylated $\beta$-catering is recognized by the $\mathrm{E} 3$ ubiquity ligase $\beta$-Trcpand is degraded by the ubiquity proteasome pathway. As a result, $\beta$-catenin in the cytoplasm remains low, unable to enter the nucleus. As a consequence, TCF/(LEF) bind to transcriptional inhibitors of the gaucho family, hat recruit histone decarboxylase to mediate transcriptional repression through chromatin compaction (8). However, how DSH (Dishevelled) phosphorylation is controlled and what DSH functions in Wnt signaling are still unknown.

\section{Non-canonic Wnt signal pathway}

The non-classical pathway is divided into the planar cell polarity (PCP) and calcium flux. During the PCP, it can be initiated by Wnt-Frizzled receptor interactions which activate DVL. DVL regulates three small GTPases including RHOA, RAC and cell division control protein 42 (CDC42) and triggers JUN N-terminal kinase (JNK), JNK activates nuclear factor (NFAT)-dependent transcription of AP1- and activated $T$ cells after entering the nucleus.

In the calcium flux, Wnt and DVL bind to each other to activate PLC which releases calcium ions. Intracellular calcium ions activate the downstream protein kinase $\mathrm{C}$ (PKC) and calcium/calmodulin-dependent protein kinase II (CaMKII) which activate nuclear factor (NFAT) (9).

\section{Wnt cooperation with other signaling pathway}

Nuclear factor kappa B (NF-кB), Stat, and $\beta$-catenindependent transcriptional activators in genes encoding in vivo plane/axis/design and stress responses in adult life Showing the most prominent performance (10). Stat is a co-activator. In advanced mammalian vertebrate hosts, $\beta$-catenin is based on Wnt and NF- $\kappa \mathrm{B} / \mathrm{Relp} 65$ pathways have become major members of Stat-associated proto- oncogenes/oncoproteins (11)

Every family member of Wnt has unique features. There are complex interactions among the $19 \mathrm{Wnt}$ members in the Wnt signaling pathway. At the same time, the Wnt signal path is closely related to other signal paths. Recently, the role of the Wntsignaling pathway in the inflammatory process began to be discovered. In addition, $\mathrm{Wnt} / \beta$-catenin pathway components can regulate inflammation and immune responses by interacting with $\mathrm{NF}-\kappa \mathrm{B}$. In turn, NF- $\kappa \mathrm{B}$ also affects the activity of the Wnt/B-catenin signaling pathway (12). Crosstalk between these two pathways can significantly affect inflammation and cancer progression. In-depth studies have found that $\mathrm{NF}-\kappa \mathrm{B}$ signaling pathway is a powerful target for the treatment of inflammatory diseases and inflammationrelated cancers, and Wnt signaling pathway can prevent or promote the development of inflammation. Another study suggests that abnormal Wnt signaling increases the risk of type 2 diabetes and Alzheimer's disease in humans during metabolic processes in organisms. GSK- $3 \beta$ kinase links Alzheimer's disease and diabetes. Therefore, it will be a potential treatment for diabetes (13). Previous studies have demonstrated that $\beta$-catenin is a negative regulator of intestinal NF- $\mathrm{\kappa B}$ activity in bacterial-induced epithelial inflammation. In the canonical pathway, Wnt binding stabilizes the transcription factor $\beta$-catenin, which in turn enters the nucleus to regulate the Wnt pathway target gene. This suggests that Wnt2 may regulate the inflammatory response by influencing signaling pathways associated with cell proliferation and apoptosis, thereby promoting host protection in the gut. Wnt2 pathways, upregulation of $\beta$-catenin, and increased viability of intestinal epithelial cells during bacterial infection (8). Members of the Wnt/ $\beta$-catenin pathway can also serve as potential therapeutic targets for many types of cancer. Further studies have shown that Stat3 is an important mediator of FZD2-mediated downstream signaling, EMT planning and cell migration. Therefore, it is possible to c develop inhibitors targeting on Wnt2 combined with Stat3 (14).

\section{Wnt/ $\beta$-catenin signaling pathway in cancer}

Mutations in oncogenes or tumor suppressor genes can lead to inappropriate activation of normal regulatory cells, resulting in uncontrolled cell proliferation and tumor formation (15). Every obstacle in any step of the Wnt pathway can cause cancer. The abnormalities can be roughly divided into three categories: one is that destruction or 
mutation of a protein, transcription factor, or gene in the Wnt signaling pathway results in the pathway being shut down or the local pathway is abnormally active. Secondly, excessive Wnt signals cause abnormal activation of the entire pathway, and cells undergo unnecessary proliferation. Thirdly, when there is no Wnt signal, other activities in the cell will stimulate or induce abnormal reactions of the cells or even the body through the Wnt pathway. The $\beta$-cateninTCF/LEF complex is a hub in the Wnt pathway. Once $\beta$-catenin is easily located in the nucleus, it can be combined with TCF/LEF to initiate the Wnt pathway (16). Structural and functional changes in the upstream components lead to degradation of $\beta$-catenin, it will cause intracellular accumulation of $\beta$-catenin (17). Cells are cancerous by pushing the cell cycle or producing abnormal proteins. There are varying degrees of $\beta$-catenin gene mutations in many tumors. Such as hepatocellular carcinoma, ovarian cancer, skin cancer, colon cancer, etc. $\beta$-catenin mutation rate can be as high as $50 \%$ or more (18).

Changes in the morphology and function of the components upstream of the Wnt pathway can also affect the state of $\beta$-catenin. Mainly including APC, GSK-3 and AXIN (19). APC is a tumor suppressor gene involved in colon cancer. The APC proteins, GSK-3 and AXIN, form a complex with $\beta$-catenin and promote phosphorylation of $\beta$-catenin. $\beta$-catenin is allowed to be degraded by proteases. Mutations in the APC gene can cause $\beta$-catenin to fail to bind the APC, and thus cannot be phosphorylated by GSK-3, so that $\beta$-catenin is blocked and accumulates in the cytosol (20). GSK can phosphorylate $\beta$-catenin which is a negative regulator, such as Wnt pathway, at the same time it is also a tumor suppressor gene. AXIN has multiple protein-protein domains and acts as a scaffold protein like APC. Detection of AXIN gene mutation in tumors such as liver cancer and colon cancer (21).

TCF is a downstream component of the Wnt pathway. In most cases it does not activate transcription. Only when bound to $\beta$-catenin, transcriptional activation occurs (22). Wnt secreted protein and its receptor FZD can also be abnormally expressed in tumors. In colon cancer and gastric cancer, the expression of FZD1/2 was found to be significantly higher than that of normal mucosa (23).

\section{Targeted drug}

The secreted Wnt protein is one of the largest families of intercellular signaling molecules in vertebrates, which plays a crucial role in embryonic development and tissue homeostasis. The Wnt gene utilizes certain forms of the transcriptional coactivator $\beta$-catenin, limiting the ability of classical genetic strategies to reveal its effects in vivo (24). Targeted drugs have small side effects and significant curative effects, which have greatly changed the treatment situation of indications, such as tumors (25). Targeted drugs are mainly divided into two categories: small molecule drugs and macromolecular monoclonal antibodies. Here we mainly focus on new drug research in the treatment of cancer with the Wnt signaling pathway. Table 1 summarized current therapeutics for Wnt pathway molecules include Porcupine inhibitors, coiled receptors and tankyrase, as well as targets for DKK1, SOST and GSK3 3 .

Tankyrase (TNKS), a key mediator of Wnt signaling, has been recognized as a novel molecular target for Wnt pathway-dependent cancers. Novel PARP inhibitor E7449 (also known as tankyrase 1 and 2), an important regulator of classical $W n t / \beta$-catenin signaling. It inhibits the enzymatic activity of PARP and additionally captures PARP1 on damaged DNA (26). In addition, E7449 stabilizes AXIN and TNKS proteins, resulting in instability of $\beta$-catenin and significantly altering the expression of Wnt target genes. E7449 enhances chemotherapy, and monotherapy has significant anti-tumor activity against breast cancer 1(BRCA 1)-deficient xenografts. Although it lacks the antitumor activity of a single drug in vivo, the pharmacodynamic effect of E7449 on Wnt target genes is observed in tumors, which is a typical finding of selective TNKS inhibitors. The antitumor activity of E7449 is increased by binding to MEK inhibition (27).

$\mathrm{Wnt} / \beta$-catenin signaling is involved in embryonic development, tissue homeostasis, and various human diseases. Abnormal activation of this pathway causes accumulation of $\beta$-catenin in the nucleus and promotes transcription of many oncogenes (28). Liang Fang and his colleagues found that a compound called LF3 strongly inhibits the abnormal binding between $\beta$-catenin and TCF-4, with little effect on healthy cells (29). In vitro experiments also confirmed that mice treated with LF3 showed a significant decrease in tumor growth and differentiation of in vivo cancer stem cells into benign lesions, while other signaling pathways other than the Wnt signaling pathway were not disturbed (30). All of these suggest that LF3 is highly promising as a lead compound and lays the foundation for the development of methods for treating human tumors that depend on the Wnt signaling pathway.

Inhibitors of Wnt production (IWPs) are known antagonists of the Wnt pathway, targeting membrane- 
Table 1 summarized current therapeutics for Wnt pathway molecules

\begin{tabular}{|c|c|c|}
\hline Target & Drug & Stage of drug development \\
\hline \multirow[t]{10}{*}{ WNT } & LGK974 & Phase I cancer \\
\hline & XNM7201 & Phase I cancer \\
\hline & CGX1321 & Phase I cancer \\
\hline & WNT974 & Preclinical cancer \\
\hline & GNF6231 & Phase I cancer \\
\hline & E7449 & Phase I cancer \\
\hline & ETC-159 & Phase I cancer \\
\hline & IWP-2 & Preclinical cancer \\
\hline & WNT-C59 & Preclinical cancer \\
\hline & OMP-54F28 & Phase I cancer \\
\hline \multirow[t]{5}{*}{ FZDs } & Vantictumab & Phase I cancer \\
\hline & $\lg \mathrm{l}-2919$ & Preclinical cancer \\
\hline & OMP-54F28 & Phase I cancer \\
\hline & OMP-131R10 & Preclinical cancer \\
\hline & OTSA101 & Phase I cancer \\
\hline \multirow[t]{6}{*}{ ROR1 } & KAN 0439834 & Preclinical cancer \\
\hline & Cirmtuzumab & Phase I cancer \\
\hline & ROR1-CD3-DART & Preclinical cancer \\
\hline & APVO425 & Preclinical cancer \\
\hline & UC-961 & Preclinical cancer \\
\hline & ROR1R-CAR-T & Preclinical cancer \\
\hline \multirow[t]{7}{*}{ AXIN } & AZ1366 & Preclinical cancer \\
\hline & G007-LK & Phase I cancer \\
\hline & NVP-TNKS656 & Preclinical cancer \\
\hline & NCB-0846 & Preclinical cancer \\
\hline & E7449 & Preclinical cancer \\
\hline & SKL2001 & Preclinical cancer \\
\hline & XAV939 & Preclinical cancer \\
\hline \multirow[t]{10}{*}{$\beta$-catenin } & BC2059 & Preclinical cancer \\
\hline & CGP049090 & Preclinical cancer \\
\hline & CWP232228 & Preclinical cancer \\
\hline & ICG-001 & Preclinical cancer \\
\hline & LF3 & Preclinical cancer \\
\hline & MSAB & Preclinical cancer \\
\hline & PKF115-584 & Preclinical cancer \\
\hline & PRI-724 & Phase II cancer \\
\hline & C-82 & Phase I cancer \\
\hline & SAH-BCL9 & Preclinical cancer \\
\hline
\end{tabular}

Table 1 (continued)
Table 1 (continued)

\begin{tabular}{lll}
\hline Target & Drug & Stage of drug development \\
\hline DKK1 & BHQ880 & Phase I cancer \\
& DKN-01 & Phase I cancer \\
& CBX7 & Preclinical cancer \\
SOST & Blosozumab & Phase I cancer \\
& BPS804 & Phase I cancer \\
& Romosozumab & Phase I cancer \\
RSPO3 & OMP-131R10 & Phase I cancer \\
DVL & NSC668036 & Preclinical cancer \\
USP & P5091 & Preclinical cancer \\
\hline
\end{tabular}

bound $\mathrm{O}$-acyltransferase, thereby preventing key Wnt ligand palmitoylation (31).

LGK974 is an effective and specific small molecule porcupine (PORCN) inhibitor. It is effective in inhibiting Wnt signaling both in vitro and in vivo, including reducing Wnt-dependent LRP6 phosphorylation and Wnt target genes. LGK974 is a new oral bioavailable cancer treatment drug in phase I clinical trials (32).

$\mathrm{DSH}$ is a positive regulator of the Wnt pathway. It binds to AXIN and the PDZ which domains in the central region. DVL binds to the carboxy terminus of the FZD receptor using the PDZ domain. The small molecule compound NSC668036 is an organic inhibitor of the PDZ domain in DVL. It can block FZD binding to PDZ, inhibits $\beta$-catenindriven gene transcription, and eliminates TGF- $\beta 1$-induced migration (33).

Dickkopf-1 (DKK-1) protein, one of the inhibitors of Wnt signaling pathway, can be competitively bound to lipoprotein receptor-associated protein 5/6 (LRP5/6), or through transmembrane protein kremen. LRP5/6 forms a ternary complex resulting in rapid endocytosis, reducing plasma membrane LRP5/6 and inhibiting the Wnt signaling pathway (34). CBX7 inhibits the $\mathrm{Wnt} / \beta$-catenin/T cytokine pathway by enhancing the expression of the $\mathrm{Wnt}$ antagonist DKK-1. In particular, CBX7 increases DKK-1 transcription by complexing with $\mathrm{p} 300$ acetyltransferase and subsequently enhancing histone acetylation of the DKK-1 promoter. Furthermore, pharmacological inhibition of DKK-1 in CBX7 overexpressing cells showed restoration of Wnt signaling $(35,36)$.

The ubiquitin-specific protease (USP) family is the largest cysteine protease. Overexpression of USP21 is associated with progression of human pancreatic ductal 
adenocarcinoma (PDAC), a PDAC oncogene. USP2 1 is capable of ubiquitination and stabilizes the TCF/LEF transcription factor TCF7, thereby promoting the dryness of cancer cells (37). Previous preclinical studies have shown that USP7 may be a potential drug target. USP7 regulates Wnt signaling catenin by deubiquitinating $\beta$-positive. P5091, a small molecule inhibitor of USP7, which can inhibit the proliferation of CRC cells and induce apoptosis in vitro. In vitro, $\mathrm{P} 5091$ inhibits proliferation of CRC cells and induces apoptosis. In the HCT116 xenograft mouse model, P5091 also inhibited tumor growth in vivo, which was consistently associated with decreased expression of $\beta$-catenin and Wnt target genes. P5091 is worthy of further development as an anticancer agent for Wnt pre-activated CRC treatment (38).

\section{Combinatorial therapeutic}

In the central nervous system (CNS), Wnt signaling has been shown to have neuroprotective effects. Conversely, its inhibition causes neurodegenerative changes, suggesting that inhibition of PORCN in cancer therapy should be used with caution and its recognition known functions may be suppressed. In addition, a study showed that the use of porcupine inhibitors for cancer treatment may increase the risk of fracture (39). Therefore, the discovery of new targets and the combined use of drugs are particularly urgent. The combination of WNT974 (formerly LGK974) and carboplatin resulted in a higher percentage of samples with a $\geq 30 \%$ reduction in ATP compared to monotherapy (31). Another study found that aspirin and LGK974 can effectively inhibit the signaling pathways of Wnt and MAPK, block cell cycle and induce apoptosis of CRC cells (40).

The combination of the PORCN inhibitor ETC-159 and the pan-PI3K inhibitor GDC-0941 enhances the inhibition of cell proliferation and glucose metabolism, and effectively inhibits the growth of RNF43 mutant pancreatic cancer xenografts in vivo. These findings indicate that dual PORCN and PI $3 \mathrm{~K} / \mathrm{mTOR}$ inhibition are potential strategies for the treatment of Wnt-driven pancreatic cancer (41).

Although tankyrase (TNKS) inhibitors have been proposed as promising candidates, many colorectal cancer models have no positive response to TNKS inhibition in vitro and in vivo. Therefore, a TNKS inhibitor (G007-LK) was used in combination with PI3K (BKM120) and EGFR (erlotinib) inhibitors (42). The data indicate that TNKS inhibitors enhance the inhibition of PI3K and EGFR in colorectal cancer cell lines (43).

XAV939 combined with 5-fluorouracil (5-FU)/cisplatin (DDP) treatment of colon cancer cells, cirmtuzumab and ibrutinib for the simultaneous treatment of leukemia cells is much more effective than treatment with either drug alone (44). The combination of ETC-159 and anti-absorbent alendronate can reduce bone loss after treatment with ETC-159 by regulating osteoclast activity and blocking the accumulation of bone marrow adipocytes (45).

A recent combination of XNM7201 and Treprizumab will be clinically tested. XNM7201 is a small molecule inhibitor of the Wnt pathway Porcupine protein. Trepril monoclonal antibody is a recombinant humanized antiPD-1 monoclonal antibody, and is the first Chinese domestic PD-1 monoclonal antibody approved for marketing (46). The combination of the two drugs is the first drug cooperation between PD-1 monoclonal antibody and Wnt inhibitor in China, and it is expected to break the long-term treatment vacancies of digestive tract tumors in the future.

\section{Conclusions}

The Wnt signaling pathway has been discovered and extensively studied for more than 30 years, and it has induced several intracellular signal transduction pathways, particularly the $W n t / \beta$-catenin-dependent pathway, the classical pathway, non-classical and $\beta$-catenin-dependent pathways. The latter include $\mathrm{Wnt} / \mathrm{Ca}^{+}$and PCP pathways (47). Wnt signaling pathway is widely involved in various processes of cancer, including tumor initiation, tumor growth, cell senescence, cell death, differentiation and metastasis (48). At present, research on the target of $\mathrm{Wnt}$ "impossible medicine" is actively carried out. In the past few years, some small molecule drugs and biological agents have entered clinical trials, and several Ib/IIa phase clinical trials of Wnt antagonists combined with cytotoxic drugs are also underway. For example, LGK974 is a new type of oral bioavailable cancer treatment drug in phase I clinical trials (49). The monoclonal antibody OMP-18R5 antagonizes Wnt ligands and inhibits the growth of many cancers and has been used in phase Ia trials of preclinical solid tumor models (50). With the gradual deepening of people's understanding of genes and their functions, the pathogenesis of many tumors has become more and more clear, which laid a good foundation for the development of targeted therapy. 


\section{Acknowledgments}

Funding: None.

\section{Footnote}

Conflicts of Interest: All authors have completed the ICMJE uniform disclosure form (available at http://dx.doi. org/10.21037/tcr.2019.12.50). The authors have no conflicts of interest to declare.

Ethical Statement: The authors are accountable for all aspects of the work in ensuring that questions related to the accuracy or integrity of any part of the work are appropriately investigated and resolved.

Open Access Statement: This is an Open Access article distributed in accordance with the Creative Commons Attribution-NonCommercial-NoDerivs 4.0 International License (CC BY-NC-ND 4.0), which permits the noncommercial replication and distribution of the article with the strict proviso that no changes or edits are made and the original work is properly cited (including links to both the formal publication through the relevant DOI and the license). See: https://creativecommons.org/licenses/by-nc-nd/4.0/.

\section{References}

1. Nusse R. The Wnt gene family in tumorigenesis and in normal development. J Steroid Biochem Mol Biol 1992;43:9-12.

2. Becker J, Wilting J. WNT Signaling in Neuroblastoma. Cancers (Basel) 2019. doi: 10.3390/cancers11071013.

3. Rodriguez-Salas N, Dominguez G, Barderas R, et al. Clinical relevance of colorectal cancer molecular subtypes. Crit Rev Oncol Hematol 2017;109:9-19.

4. Tran FH, Zheng JJ. Modulating the wnt signaling pathway with small molecules. Protein Sci 2017;26:650-61.

5. Logan CY, Nusse R. The Wnt signaling pathway in development and disease. Annu Rev Cell Dev Biol 2004;20:781-810.

6. Zhan T, Rindtorff N, Boutros M. Wnt signaling in cancer. Oncogene 2017;36:1461-73.

7. Bravo DT, You L, Mazieres J, et al. Targeting Wnt-2 in Mesothelioma and Lung Cancer. Clinical Lung Cancer 2008;9:289.

8. Kimura M, Nakajima-Koyama M, Lee J, et al. Transient Expression of WNT2 Promotes Somatic Cell Reprogramming by Inducing beta-Catenin Nuclear
Accumulation. Stem Cell Reports 2016;6:834-43.

9. Kahn M. Can we safely target the WNT pathway? Nat Rev Drug Discov 2014;13:513-32.

10. Sinkovics JG. The cnidarian origin of the proto-oncogenes NF-kappaB/STAT and WNT-like oncogenic pathway drives the ctenophores (Review). Int J Oncol 2015;47:1211-29.

11. Liu X, Lu R, Wu S, et al. Wnt2 inhibits enteric bacterialinduced inflammation in intestinal epithelial cells. Inflamm Bowel Dis 2012;18:418-29.

12. $\mathrm{Pu}$ P, Zhang Z, Kang C, et al. Downregulation of Wnt2 and beta-catenin by siRNA suppresses malignant glioma cell growth. Cancer Gene Ther 2009;16:351-61.

13. Yang S, Liu Y, Li MY, et al. FOXP3 promotes tumor growth and metastasis by activating $\mathrm{Wnt} /$ beta-catenin signaling pathway and EMT in non-small cell lung cancer. Mol Cancer 2017;16:124.

14. Zhuang X, Zhang H, Li X, et al. Differential effects on lung and bone metastasis of breast cancer by Wnt signalling inhibitor DKK1. Nat Cell Biol 2017;19:1274-85.

15. Baron R, Kneissel M. WNT signaling in bone homeostasis and disease: from human mutations to treatments. Nat Med 2013;19:179-92.

16. Cadigan KM, Waterman ML. TCF/LEFs and Wnt signaling in the nucleus. Cold Spring Harb Perspect Biol 2012. doi: 10.1101/cshperspect.a007906.

17. Hrckulak D, Kolar M, Strnad H, et al. TCF/LEF Transcription Factors: An Update from the Internet Resources. Cancers (Basel) 2016. doi: 10.3390/cancers8070070.

18. Lee H, Kim N, Yoo YJ, et al. beta-catenin/TCF activity regulates IGF-1R tyrosine kinase inhibitor sensitivity in colon cancer. Oncogene 2018;37:5466-75.

19. Stamos JL, Weis WI. The beta-catenin destruction complex. Cold Spring Harb Perspect Biol 2013;5:a007898.

20. Valvezan AJ, Zhang F, Diehl JA, et al. Adenomatous polyposis coli (APC) regulates multiple signaling pathways by enhancing glycogen synthase kinase-3 (GSK-3) activity. J Biol Chem 2012;287:3823-32.

21. Zhu B, Wang DN, Gong XM, et al. Expression of AXIN and MACC1 in Gastric Carcinoma and Its Clinical Significance. Sichuan Da Xue Xue Bao Yi Xue Ban 2018;49:59-64.

22. Bhardwaj D, Nager M, Camats J, et al. Chemokines induce axon outgrowth downstream of Hepatocyte Growth Factor and TCF/beta-catenin signaling. Front Cell Neurosci 2013;7:52.

23. Tao L, Zhang J, Meraner P, et al. Frizzled proteins are colonic epithelial receptors for C. difficile toxin B. Nature 2016;538:350-5.

24. Liu S, Chen X, Chen R, et al. Diagnostic role of Wnt 
pathway gene promoter methylation in non small cell lung cancer. Oncotarget 2017;8:36354-67.

25. Steinhagen H. Igniting Small-Molecule Drug Discovery. ChemMedChem 2016;11:148-9.

26. Bitler BG, Watson ZL, Wheeler LJ, et al. PARP inhibitors: Clinical utility and possibilities of overcoming resistance. Gynecol Oncol 2017;147:695-704.

27. McGonigle S, Chen Z, Wu J, et al. E7449: A dual inhibitor of PARP1/2 and tankyrase1/2 inhibits growth of DNA repair deficient tumors and antagonizes Wnt signaling. Oncotarget 2015;6:41307-23.

28. Shang S, Hua F, Hu ZW. The regulation of beta-catenin activity and function in cancer: therapeutic opportunities. Oncotarget 2017;8:33972-89.

29. Yan M, Li G, An J. Discovery of small molecule inhibitors of the Wnt/beta-catenin signaling pathway by targeting beta-catenin/Tcf4 interactions. Exp Biol Med (Maywood) 2017;242:1185-97.

30. Fang L, Zhu Q, Neuenschwander M, et al. A SmallMolecule Antagonist of the beta-Catenin/TCF4 Interaction Blocks the Self-Renewal of Cancer Stem Cells and Suppresses Tumorigenesis. Cancer Res 2016;76:891-901.

31. Boone JD, Arend RC, Johnston BE, et al. Targeting the Wnt/beta-catenin pathway in primary ovarian cancer with the porcupine inhibitor WNT974. Lab Invest 2016;96:249-59.

32. Guimaraes PPG, Tan M, Tammela T, et al. Potent in vivo lung cancer Wnt signaling inhibition via cyclodextrin-LGK974 inclusion complexes. J Control Release 2018;290:75-87.

33. Wang C, Dai J, Sun Z, et al. Targeted inhibition of disheveled PDZ domain via NSC668036 depresses fibrotic process. Exp Cell Res 2015;331:115-22.

34. Zhang ZC, Liu JX, Shao ZW, et al. In vitro effect of microRNA-107 targeting Dkk-1 by regulation of Wnt/ beta-catenin signaling pathway in osteosarcoma. Medicine (Baltimore) 2017;96:e7245.

35. Ni SJ, Zhao LQ, Wang XF, et al. CBX7 regulates stem celllike properties of gastric cancer cells via p16 and AKT-NFkappaB-miR-21 pathways. J Hematol Oncol 2018;11:17.

36. Jiang F, Yang X, Meng X, et al. Effect of CBX7 deficiency on the socket healing after tooth extractions. J Bone Miner Metab 2019;37:584-93.

37. Hou P, Ma X, Zhang Q, et al. USP21 deubiquitinase promotes pancreas cancer cell stemness via Wnt pathway activation. Genes Dev 2019;33:1361-6.

38. An T, Gong Y, Li X, et al. USP7 inhibitor P5091 inhibits Wnt signaling and colorectal tumor growth. Biochem Pharmacol 2017;131:29-39.
39. Funck-Brentano T, Nilsson KH, Brommage R, et al. Porcupine inhibitors impair trabecular and cortical bone mass and strength in mice. J Endocrinol 2018;238:13-23.

40. Bagheri M, Tabatabae Far MA, Mirzaei H, et al. Evaluation of antitumor effects of aspirin and LGK974 drugs on cellular signaling pathways, cell cycle and apoptosis in colorectal cancer cell lines compared to oxaliplatin drug. Fundam Clin Pharmacol 2019.

41. Zhong Z, Sepramaniam S, Chew XH, et al. PORCN inhibition synergizes with PI3K/mTOR inhibition in Wnt-addicted cancers. Oncogene 2019;38:6662-77.

42. Liu $X, W u$ H, Huang P, et al. JQ1 and PI3K inhibition synergistically reduce salivary adenoid cystic carcinoma malignancy by targeting the c-Myc and EGFR signaling pathways. J Oral Pathol Med 2019;48:43-51.

43. Solberg NT, Waaler J, Lund K, et al. TANKYRASE Inhibition Enhances the Antiproliferative Effect of PI3K and EGFR Inhibition, Mutually Affecting beta-CATENIN and AKT Signaling in Colorectal Cancer. Mol Cancer Res 2018;16:543-53.

44. Yu J, Chen L, Cui B, et al. Cirmtuzumab inhibits Wnt5ainduced Rac1 activation in chronic lymphocytic leukemia treated with ibrutinib. Leukemia 2017;31:1333-9.

45. Madan B, McDonald MJ, Foxa GE, et al. Bone loss from Wnt inhibition mitigated by concurrent alendronate therapy. Bone Res 2018;6:17.

46. Iwai Y, Hamanishi J, Chamoto K, et al. Cancer immunotherapies targeting the PD-1 signaling pathway. J Biomed Sci 2017;24:26.

47. Tai D, Wells K, Arcaroli J, et al. Targeting the WNT Signaling Pathway in Cancer Therapeutics. Oncologist 2015;20:1189-98.

48. Pai SG, Carneiro BA, Mota JM, et al. Wnt/beta-catenin pathway: modulating anticancer immune response. J Hematol Oncol 2017;10:101.

49. Yang JM, Huang HM, Cheng JJ, et al. LGK974, a PORCUPINE inhibitor, mitigates cytotoxicity in an in vitro model of Parkinson's disease by interfering with the WNT/ beta-CATENIN pathway. Toxicology 2018;410:65-72.

50. Pavlovic Z, Adams JJ, Blazer LL, et al. A synthetic antiFrizzled antibody engineered for broadened specificity exhibits enhanced anti-tumor properties. MAbs 2018;10:1157-67.

Cite this article as: Zhang Y, Zu D, Chen Z, Ying G. An update on Wnt signaling pathway in cancer. Transl Cancer Res 2020;9(2):1246-1252. doi: 10.21037/tcr.2019.12.50 